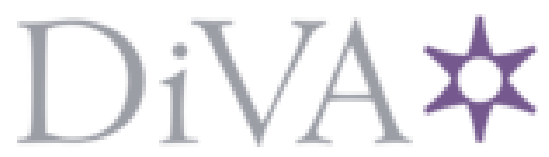

http://www.diva-portal.org

This is the published version of a paper published in Lab on a Chip.

Citation for the original published paper (version of record):

Carlborg, C., Gylfason, K., Kazmierczak, A., Dortu, F., Banuls Polo, M. et al. (2010)

A packaged optical slot-waveguide ring resonator sensor array for multiplex label-free assays in labs-on-chips.

Lab on a Chip, 10(3): 281-290

http://dx.doi.org/10.1039/b914183a

Access to the published version may require subscription.

N.B. When citing this work, cite the original published paper.

Permanent link to this version:

http://urn.kb.se/resolve?urn=urn:nbn:se:kth:diva-12208 


\title{
A packaged optical slot-waveguide ring resonator sensor array for multiplex label-free assays in labs-on-chips $\dagger$
}

\author{
C. F. Carlborg, $\star^{* a}$ K. B. Gylfason, $\leftarrow^{a}$ A. Kaźmierczak, ${ }^{b}$ F. Dortu, ${ }^{b}$ M. J. Bañuls Polo, ${ }^{c}$ A. Maquieira Catala, ${ }^{c}$ \\ G. M. Kresbach, ${ }^{d}$ H. Sohlström, ${ }^{a}$ T. Moh, ${ }^{a}$ L. Vivien, ${ }^{e}$ J. Popplewell,${ }^{f}$ G. Ronan,${ }^{f}$ C. A. Barrios,${ }^{g}$ G. Stemme ${ }^{a}$ \\ and W. van der Wijngaart ${ }^{a}$
}

\author{
Received 15th July 2009, Accepted 29th September 2009 \\ First published as an Advance Article on the web 12th November 2009 \\ DOI: $10.1039 / b 914183 a$
}

\begin{abstract}
We present the design, fabrication, and characterisation of an array of optical slot-waveguide ring resonator sensors, integrated with microfluidic sample handling in a compact cartridge, for multiplexed real-time label-free biosensing. Multiplexing not only enables high throughput, but also provides reference channels for drift compensation and control experiments. Our use of alignment tolerant surface gratings to couple light into the optical chip enables quick replacement of cartridges in the readout instrument. Furthermore, our novel use of a dual surface-energy adhesive film to bond a hard plastic shell directly to the PDMS microfluidic network allows for fast and leak-tight assembly of compact cartridges with tightly spaced fluidic interconnects. The high sensitivity of the slot-waveguide resonators, combined with on-chip referencing and physical modelling, yields a volume refractive index detection limit of $5 \times 10^{-6}$ refractive index units (RIUs) and a surface mass density detection limit of $0.9 \mathrm{pg} \mathrm{mm}^{-2}$, to our knowledge the best reported values for integrated planar ring resonators.
\end{abstract}

\section{Introduction}

The measurement of the optical properties of liquid samples is one of the cornerstones of analytical chemistry. Optical absorption spectroscopy, for example, allows probing of the chemical bonds present in a sample, and real-time refractive index measurement enables label-free study of binding dynamics. Accordingly, to bring these powerful analytical tools into the hands of a wider user base, there is a strong interest in integrating optical sensors in labs-on-chips. Even though an abundance of optical sensor principles has been demonstrated, very few have successfully been integrated in complete labs-on-chips.

In this work, we present the design, fabrication, and characterisation of a packaged array of optical refractive index sensors, integrated with microfluidic sample handling in a compact cartridge, for real-time label-free biosensing. In particular, we address three important aspects of optical labs-on-chips: (1) onchip multiplexing of the optical measurement for higher throughput and referencing, (2) the chip packaging into

\footnotetext{
${ }^{a}$ Microsystem Technology Laboratory, KTH-Royal Institute of Technology, Osquldas väg 10, SE-10044 Stockholm, Sweden. E-mail: fredrik.carlborg@ee.kth.se; Fax: +468100 858; Tel: +468790 7794

${ }^{b}$ Multitel a.s.b.l., B-7000 Mons, Belgium

${ }^{c}$ Departamento de Quimica, Universidad Politécnica de Valencia, 46022 Valencia, Spain

${ }^{d}$ Zeptosens-A Division of Bayer (Schweiz) AG, CH-4108 Witterswil, Switzerland

'Institut d'Electronique Fondamentale, Université Paris-Sud 11, 91405 Orsay, France

${ }^{f}$ Farfield Group Ltd, Cheshire, UK CW1 $6 G U$

${ }^{g}$ Instituto de Sistemas Optoelectrónicos y Microtecnología, Universidad Politécnica de Madrid, 28040 Madrid, Spain

$\dagger$ Electronic supplementary information (ESI) available: Supplementary figures (S1, S2, S3). See DOI: 10.1039/b914183a

\$ These two authors contributed equally to this work.
}

a cartridge, and (3) the alignment of the chip to the read-out instrument.

In the first section below we give background on each of the three aspects mentioned above and explain how we improve on each of them. Next, we describe the design and fabrication of the sensor cartridge parts and how they are combined using our novel packaging method. We then proceed with a description of the necessary peripheral equipment, followed by the measurement principle. In the experimental section we detail the volume and surface sensing experiments. Finally, we discuss the results, and provide a summary and conclusion.

\section{Background and advances in this work}

The advantages of scaling analytical chemical and biological instruments down to a single chip have been extensively explored in recent reviews. ${ }^{1}$ The advantages include: automation of the analysis, increased mobility of the instrument, shorter response times, reduced manual sample handling, and a low cost per test. On the other hand, downscaling the instrument limits the space available for active temperature control components. Therefore, space consuming environmental control should be replaced by on-chip referencing and compensation techniques.

\section{Integration of multiple sensors for parallel operation and on-chip} referencing

To leverage the full potential of optical analysis in labs-on-chips, their design should allow for parallel operation of multiple optical transducers. Parallel operation not only yields higher throughput by multiple analyses of one sample, or simultaneous analyses of multiple samples, but also, more importantly, it provides reference channels for drift compensation and control experiments. Such reference measurements are particularly 
important for automated labs-on-chips and chips without temperature stabilisation.

The micro-fabrication technology developed by the microelectronics industry provides the means to efficiently replicate devices over a full silicon wafer. These techniques can equally well be used to create integrated optical circuits. One type of integrated optical sensor, that has recently been under intense investigation for on-chip label-free detection, is the planar waveguide ring resonator. ${ }^{2-5}$ Due to its small footprint and ease of integration with other on-chip optical and fluidic functions, the ring resonator is a particularly interesting optical sensor for labs-on-chips.

In ring resonators, light propagates in the form of circulating waveguide modes. The circulating waves add constructively at those wavelengths that are divisors of the ring circumference. These are referred to as the ring resonance wavelengths.

Even though the light is guided in the high refractive index waveguide core, by total internal reflection at the boundary between the core and the low index material that surrounds it, a fraction of the light, known as the evanescent field, extends out from the core. The evanescent field extends a few hundred nanometres into the surrounding media, and thus the refractive index of a sample close to the core surface influences the propagation of light in the ring. In contrast to Mach-Zehnder waveguide interferometers, the degree of interaction of light and sample in ring resonators is not limited by the physical length of the sensing waveguide, but rather by the number of revolutions of light in the resonator, characterised by the quality factor $Q$.

Ring resonators are essentially refractive index sensors, but since the refractive index of aqueous protein solutions is linear with density ${ }^{6}$ they can also be used to measure the mass of protein binding on their surface. Thus, depending on whether the surface of the ring is functionalised with specific chemical receptors, ring resonators can be used either for non-specific volume refractive index sensing or specific surface sensing. Labelfree surface sensing with single planar ring resonators has been accomplished by multiple groups. ${ }^{3,4,7}$

However, multiplexing of several ring resonator sensors on a single chip, integrated with microfluidics, has, to our knowledge, not been reported previously. Recently, five ring sensors were integrated on a single chip, each connected to individual input and output optical fibres ${ }^{5}$ but no fluidics were integrated and light splitting was handled off-chip. Multiplex operation of ring resonators made in glass capillaries has been suggested, but so far only multiple detection of the same sample has been shown. ${ }^{8}$

The sensitivity of waveguide ring resonators, that is the shift of resonance wavelength per refractive index or mass unit, depends on the extent of overlap of the evanescent field with the sample., ${ }^{49}$ As illustrated by the waveguide cross-section in Fig. S1, $\dagger$ most of the optical power in conventional strip waveguides propagates in the solid waveguide core, while only a small fraction propagates in the liquid sample. The overlap can be increased by using optical slot waveguides - a recent development in the field of integrated optics. ${ }^{10}$ A slot-waveguide consists of two rails of a high index material separated by a low index slot region of subwavelength width. With proper design, this double core structure acts as one waveguide and supports only the lowest order transverse electric (TE) and transverse magnetic (TM) modes, with a considerable fraction of the power of the TE mode propagating in the low index slot.

We have previously reported on volume refractive index sensing with a single slot-waveguide ring resonator. ${ }^{11}$ The reported sensitivity of $212 \mathrm{~nm}$ resonance wavelength shift per refractive index unit (RIU) is three times that of a recently reported conventional strip waveguide ring resonator sensor. ${ }^{4}$ Furthermore, we have demonstrated the utility of slot-waveguide ring resonators for label-free surface sensing with a detection limit of $28 \mathrm{pg} \mathrm{mm}^{-2}$ for bovine serum albumin antibody (antiBSA) captured on the waveguide surface. ${ }^{12}$

In this work, we integrate several slot-waveguide ring resonators with microfluidics on a chip, and characterise the sensor chip by multiplex volume and surface sensing experiments.

\section{Sample handling and packaging}

One of the prerequisites for portable analysis platforms is the integration of sample handling on-chip in order to reduce size and simplify the analysis. To this end, manual sample and reagent handling are replaced by a microfluidic network that precisely handles sub-microlitre volumes.

In the design of microfluidic networks it is important to consider the mass transport of analyte to the transducer in order to increase its dynamic range towards fast reaction kinetics. ${ }^{13}$ When free analyte binds to the transducer surface it needs to be replenished from the bulk solution. If the binding reaction is faster than the diffusion of analyte down to the transducer, the reaction rate will be limited by the diffusional mass transport of analyte down to the sensor. This will lead to a slower response of the transducer and risk of underestimating the reaction rate of the binding reaction. The relation between diffusion and binding kinetics is defined by the Damköhler number, Da $=k_{\mathrm{on}} C_{\mathrm{s} 0} h / D$, where $k_{\mathrm{on}}$ is the association rate of the binding reaction, $C_{\mathrm{s} 0}$ is the surface concentration of binding sites, $h$ is the height of the reaction chamber and $D$ is the diffusion constant of the analyte. If $\mathrm{Da} \gg 1$, the reaction will be completely limited by diffusion.

Advective transport of the analyte over the transducer is often required to lift the diffusion limitation. If the convection of analyte is faster than the diffusion, the concentration gradient will be small at the transducer surface, and the binding reaction rate will dominate the dynamics of the transducer read-out. The relation between advection and diffusion of the analyte is defined by the Péclet number, $\mathrm{Pe}=U h / D$, where $U$ is the average flow speed, $h$ is the height of the channel and $D$ is the diffusion constant. If $\mathrm{Pe} \gg 1$, advection dominates the flow and the concentration gradient at the surface is small.

To accurately determine dynamics for fast reactions and to speed up the time-to-result, diffusion limited micro-wells are not sufficient and a microfluidic network is required to provide the advective transport. Moreover, to be able to run several different samples simultaneously and reference the different transducers to each other we need a multichannel network where single transducers can be individually addressed.

Soft lithography is an established method for high precision fabrication of such microfluidic channels in soft polymers. The most commonly used material is poly(dimethylsiloxane) (PDMS), a bio-compatible, transparent, rubber-like polymer in which features can be replicated down to nanometre 
dimensions. ${ }^{14}$ Furthermore, a clean room is not needed for the casting process, which helps keeping the cost low.

The properties of PDMS allow precise control of the liquid front in pressure driven flows. The polymer surface can be activated in an oxygen plasma to expose hydroxyl groups $(-\mathrm{OH})$ that can form covalent siloxane bonds ( $\mathrm{Si}-\mathrm{O}-\mathrm{Si}$ ) when brought in contact with silicon or glass substrates. ${ }^{15}$ However, many of the PDMS properties are unsuitable as a protective packaging material for a cartridge, ${ }^{16}$ in particular it deforms easily under pressure. A hard outer shell is therefore typically needed. Unfortunately, the rubber-like, low-energy surface of PDMS adheres poorly to many materials used in packaging, such as poly(methyl methacrylate) (PMMA) or poly(urethane), even with plasma activation. Unpatterned PDMS can be bonded to PMMA using hot embossing and heating over the glass transition temperature of PMMA,${ }^{17}$ but this will cause deformation of the PMMA.

Because of its simplicity, the prevalent packaging solution for labs-on-chips based on PDMS or other rubber-like polymers is to clamp the soft polymer layer between the sensor chip and a hard plastic shell. The amount of clamping is then often adjusted with precision screws. ${ }^{4}$ Although this method is flexible, it is limited to low liquid throughput, because of the leakage risk at high pressure between the PDMS and the hard plastic, in particular at tightly spaced fluid ports. There is also a risk that shallow channels in the soft material will be blocked if the package is unevenly clamped. A direct bonding method, via a thin intermediate adhesive layer without high temperature curing, would be less complicated, ensure leak-tightness, and eliminate the blocking risk.

Recently, a PDMS/tape composite was presented ${ }^{18}$ that combines the attractive properties of PDMS with the adhesive flexibility of acrylic double sided tape. However, it requires an intermediate thin layer of PDMS to be spun and cured on the tape to enable adhesion to the bulk PDMS component. The reason is that the acrylic glue adheres poorly to rubbery, lowenergy surface materials such as PDMS.

In this work we solve the adhesion problem to the packaging material by introducing a dual surface-energy adhesive film, where one side of the film adheres directly to the PDMS and the other to a hard plastic shell used to package the chip.

\section{Coupling of light into labs-on-chips and alignment with read-out instrument}

To limit chip cost and complexity, optical sensors in labs-onchips usually rely on off-chip light sources and detectors. Thus, a complete measurement system must include some means of coupling light in and out of the chip. Because of the sub-micrometre cross-sectional dimensions of the single-mode waveguides used in ring resonators, coupling light into the chip is more challenging than coupling it out, and providing enough light to each of the integrated resonators becomes ever more challenging as their numbers grow.

Optical fibres are well suited to transport light from an off-chip source to the packaged chip, and light can be coupled into a planar waveguide by aligning a cut single-mode fibre to it at the chip edge, as illustrated in Fig. S2(A). $\dagger$ However, since the $7 \mu \mathrm{m}$ mode diameter of a single-mode fibre is much larger than the sub-micrometre mode diameter of the on chip waveguide, only a small fraction of the fibre mode will couple into the waveguide. There are two ways to improve the coupling efficiency: reduce the mode diameter of the incoming beam at the edge to match that of the waveguide on-chip, or expand the mode diameter of the waveguide at the edge to match that of the fibre.

The first option has been implemented with lensed fibres that focus the transmitted beam onto the waveguide end face. ${ }^{11}$ This solution is, however, not practical for user replaceable cartridges, since sub-micrometre alignment tolerances cannot be kept between cartridge replacements. Furthermore, labour intensive polishing of the input waveguide end face is required for efficient coupling, thus effectively negating any potential cost benefits of mass production with silicon micro-fabrication technology.

Surface grating couplers can be used to exploit the second option. The mode is expanded in the plane of the chip surface by a waveguide taper, as illustrated in Fig. S2(B).† A suitably designed grating, etched into the expanded waveguide section alters the propagation direction of the light, allowing the input fibre, or free space optics, to be positioned almost perpendicular to the surface. With the effective coupling length of the grating adjusted to the size of the mode field from the fibre, it also matches the out-of-plane mode sizes. In this arrangement, the overlap of the waveguide mode and the fibre mode is much larger, providing an improved coupling efficiency. More importantly, since the area of overlap has been scaled up quadratically, the alignment tolerances have been greatly relaxed.

In this work, we use a fully etched input grating designed for a high coupling efficiency, a large coupling angle tolerance, and simple fabrication. ${ }^{19}$ The less critical out-coupling is done by imaging the waveguide end faces at a chip edge onto a one dimensional (1D) photodiode array. No polishing is required for the fabrication of the chips and the compact cartridge can be quickly inserted into the read-out instrument and automatically aligned by a two dimensional translation stage.

\section{Design, fabrication, and assembly of the sensor cartridge}

The novel sensor cartridge design, utilising the dual surfaceenergy adhesive film bonding method, is shown in Fig. 1. The exploded view of the system shows the sensor cartridge positioned on the alignment platform of the read-out instrument. The cartridge is a stack of 4 bonded layers: the optical chip rests on a temperature controlled alignment platform in the read-out instrument and aligns to three pins protruding from it. The microfluidic distribution layer supplies each optical transducer site with sample. It is bonded directly to the optical chip surface and to the hard plastic shell by an intermediate adhesive film. Light from a tunable laser is coupled into the optical chip from above and collected from the long edge of the chip by imaging the cut end faces of the 8 output waveguides on a 1D photodiode array. Fluidic ports for sample injection are formed by steel tubes glued into the hard plastic shell.

\section{Optical chip}

The optical chip consists of a silicon substrate, with the integrated optical components etched into a silicon nitride thin-film, 


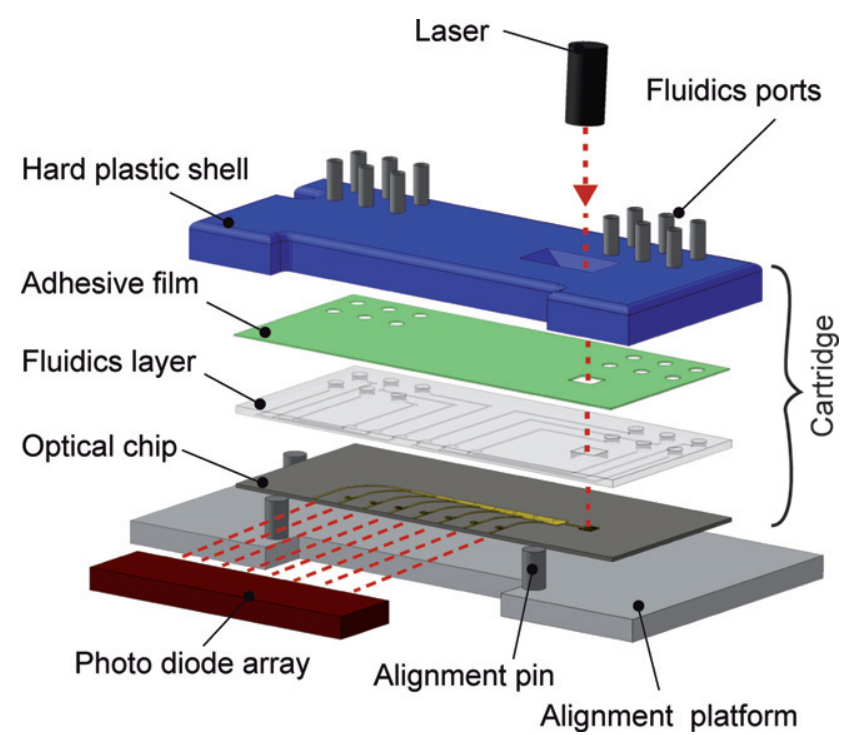

Fig. 1 A schematic exploded view of the sensor cartridge above the alignment platform, exposing the 4 permanently bonded layers of the cartridge: the optical chip, the microfluidic layer, the adhesive film, and the hard plastic shell. Cutouts in the hard plastic shell free the edge of the precision cut silicon optical chip for accurate alignment against 3 pins protruding from the alignment platform of the read-out instrument. Light is coupled in from the top via a surface grating coupler, and collected at the long edge of the optical chip by imaging the output facets on a $1 \mathrm{D}$ InGaAs photodiode array. Fluidic ports for sample injection are formed by steel tubes glued into the hard plastic shell.

embedded in a silicon dioxide cladding on its surface. Since silicon nitride is nearly impervious to diffusion of moisture and sodium ions,${ }^{20}$ it maintains a stable refractive index when operating in biological liquids, making it a suitable material for waveguide sensors. Additionally, silicon oxide and nitride have a high refractive index contrast $(\Delta n=0.5)$, thus permitting close spacing of integrated optical components, by allowing small ring and bend radii without excessive bending losses. The choice of laser wavelength is a trade-off between a narrow slot-width at short wavelengths, that may limit analyte access, and light absorption in water at longer wavelengths, that limits the resonator quality factor. Our choice of the standard telecommunication wavelength of $1310 \mathrm{~nm}$ is a good compromise and ensures that a wide selection of laser sources is available.

Fig. 2 is a top view of the layout of the optical circuit, occupying a chip area of $3 \times 7 \mathrm{~mm}^{2}$. Light enters from above via the surface grating coupler $(\mathrm{C})$. The propagating light is then split, by a multi-mode interference splitter (B) ${ }^{21}$ into eight channels: channel REF1, which has no sensor and is used for alignment and laser amplitude compensation; channel REF2, which is coupled to a reference slot-waveguide ring resonator covered with silicon dioxide cladding; and channels M1 to M6, containing the transducer sites, where openings have been etched in the silicon dioxide top cladding to allow for liquid sample access down to the slot-waveguide ring resonators (A). The orientation of the input grating and splitter, perpendicular to the output edge, ensures that stray light is not directed to the detector array.

The optical components of the sensor chip are fabricated by standard silicon micro-fabrication methods. First, a $3.26 \mu \mathrm{m}$ thick bottom cladding is grown by thermal oxidation of the silicon substrate and a $300 \mathrm{~nm}$ silicon nitride device layer deposited on top by low pressure chemical vapour deposition (LPCVD). Next, the device layer is patterned by electron beam lithography and dry etching. A negative electron beam resist (ma-N 2403, Micro Resist Technology, Germany) is particularly well suited for patterning narrow optical waveguides, since only a small fraction of the surface needs to be exposed. For mass production, standard deep ultra-violet lithography could be used. ${ }^{22}$ The patterned waveguides are then covered by a $530 \mathrm{~nm}$ thick top cladding layer of silicon dioxide deposited by tetraethyl orthosilicate (TEOS) based LPCVD and openings down to the 6 transducer sites are patterned by optical lithography and wet etching in buffered hydrofluoric acid. The thicknesses of the top and bottom oxide cladding layers are chosen for maximum coupling efficiency of the input grating coupler. ${ }^{19}$

The process is concluded by dicing the chips to size. By employing inverted waveguide tapers ${ }^{23}$ to limit the divergence the 8 output beams, in addition to a proper choice of a lens and a detector array in the output optics, ${ }^{24}$ we eliminate the need for polishing the output edge. This is of great importance for mass production, since costly manual handling of individual chips is avoided.

\section{Microfluidic distribution layer}

The microfluidic distribution layer, shown in Fig. 3(C), is manufactured in PDMS by standard soft lithography methods. ${ }^{14}$ The molding master is fabricated in SU-8 2015 (MicroChem, USA) by UV-lithography. The PDMS prepolymer (Sylgard 184, Dow Corning, USA) is cast on the master to a thickness of $2 \mathrm{~mm}$, degassed to remove air bubbles, and cured at $65^{\circ} \mathrm{C}$ for $2 \mathrm{~h}$ in an oven. The fluidic structure is then cut out, and trough holes are punched for liquid input and output ports as well as for laser access. Each of the six sensors (M1-M6) is individually addressable by a separate fluidic channel, with cross-sections of $200 \times 20 \mu \mathrm{m}^{2}$ at the widest section over the transducer. Assuming a typical diffusion constant for proteins of $D=10^{-10} \mathrm{~m}^{2} \mathrm{~s}^{-1}$, a flow rate of $10 \mu \mathrm{min}^{-1}$, and using a channel height of $20 \mu \mathrm{m}$, the Péclet number is 8340 , which indicates that advection dominates over diffusion. This ensures that we can measure fast binding dynamics accurately.

The six channels require twelve input/output ports, which are distributed with a standard $3 \mathrm{~mm}$ microwell plate pitch. The microchannels widen over each transducer (Fig. 3(B)), to relax the alignment tolerance enough to allow alignment of the microfluidic layer with the optical chip under a microscope. When brought in contact with the clean oxide surface of the chip, a leak-tight bond is formed.

\section{Intermediate adhesive bonding layer}

Because of the poor adhesion between the rubber-like PDMS and hard plastics such as PMMA, we developed a bonding method using a dual surface-energy adhesive film (5302A, Nitto Denko, Japan). The side facing the PDMS has a silicon based pressure sensitive adhesive that bonds to rubber-like, low-energy surfaces such as PDMS. The side facing the hard plastic shell has a standard acrylic pressure sensitive adhesive that easily seals to high-energy surfaces. The thickness of the adhesive film is $85 \mu \mathrm{m}$. 


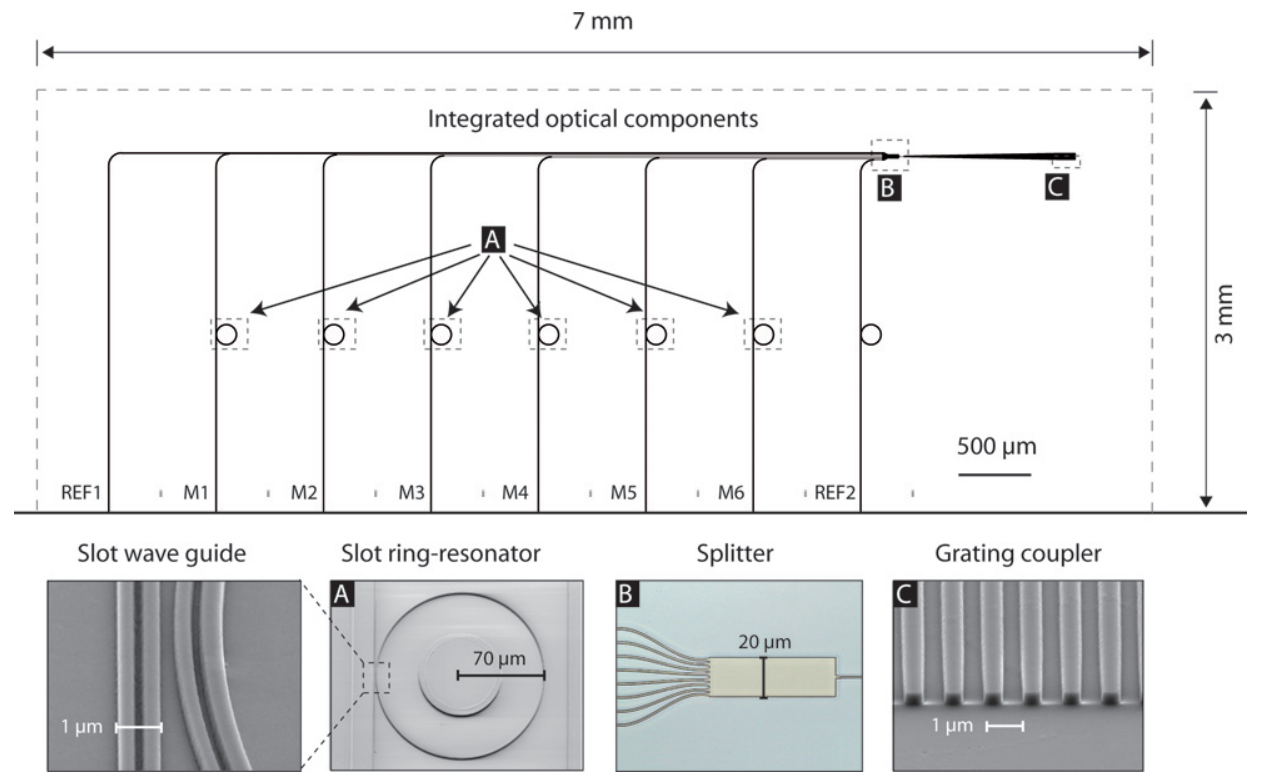

Fig. 2 A top view of the layout of the optical chip: light is injected at the surface grating coupler (C) and split by the multi-mode interference splitter (B) to the six transducer channels M1-M6 and the two reference channels REF1 and REF2. Insets are an optical micrograph of the splitter (B); electron micrographs of the grating coupler (C); and a slot-waveguide ring resonator (A), with an enlargement of the coupling region.

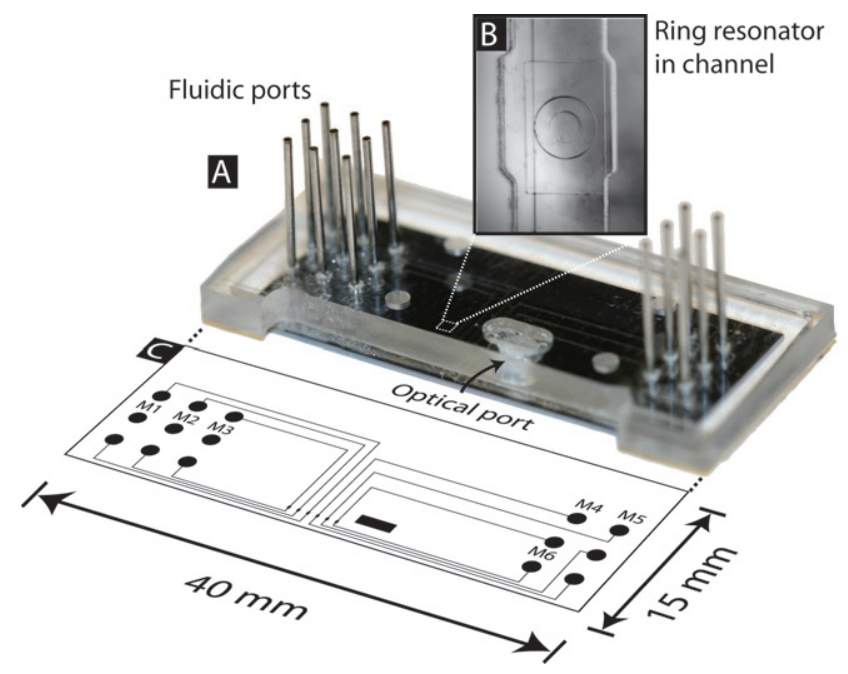

Fig. 3 (A) A photograph of one of the fabricated cartridges. The steel tubes glued to the hard plastic shell provide a stable fluidic interface to the microfluidic network below. Only twelve of the fifteen access ports were used in the current design. At the front long edge, the through hole for laser access down to the optical chip is visible. The edge of the optical chip itself can also be seen in the cutout region of the hard plastic shell. (B) The inset shows one of the ring resonators in its fluidic channel. (C) The fluidic layout with individual channel to each sensor (M1-M6).

The adhesive film is cut to the dimensions of the PDMS layer and through holes are manually punched to match the input and output ports. The film is then sandwiched between the hard plastic shell and the microfluidic layer.

The bond was tested by pressurising the finalised cartridge with up to 1 bar of air pressure without leakage at the interfaces or cross-talk between the channels. The dual surfaceenergy tape allows fast assembly without liquid glue or plasma treatment.

\section{Hard plastic shell}

The final component of the cartridge is a hard PMMA shell (Fig. 3) that protects both the soft PDMS layer and the brittle optics chip. It also provides an interface for the fluidic and optical inputs and outputs. The shell is shaped and through holes drilled, with a computer controlled milling machine (CNC). To ensure precise alignment of the optical chip to the read-out instrument, cutouts for alignment pins are milled into the front edge, and one of the short edges of the PMMA to allow physical contact between the pins and the precision cut silicon chip. Steel tubing for interfacing with the fluidic network is glued into the through holes.

\section{Read-out instrument}

The read-out instrument consists of the peripheral equipment required to operate the sensor cartridge. As shown in the block diagram in Fig. 4, the read-out instrument has three main functions: the control of liquids, light, and temperature.

Liquid flow is controlled by syringe pumps (TSE 540060, TSE Systems, Germany), one for each flow channel. During operation, the pumps supply a continuous flow of buffer to the chip, and samples are injected into the flow using in-line injection valves (Rheodyne 9725i, IDEX Health and Science, USA).

Light from a mechanically tuned external cavity laser (TSL$210 \mathrm{~V}$, Santec, Japan) is coupled into the chip from free space optics above the surface. The minimum wavelength step of the laser is $1 \mathrm{pm}$ and the tuning range is $1260-1360 \mathrm{~nm}$. In general fast laser wavelength sweeping is preferred since noise can be reduced by averaging over many scans, and for measuring very fast binding reactions, a faster, electronically tuned source is necessary. As seen in Fig. 1, the cartridge is aligned on an aluminium platform with three steel pins protruding from its surface. Since the pins register directly on the precision cut silicon 


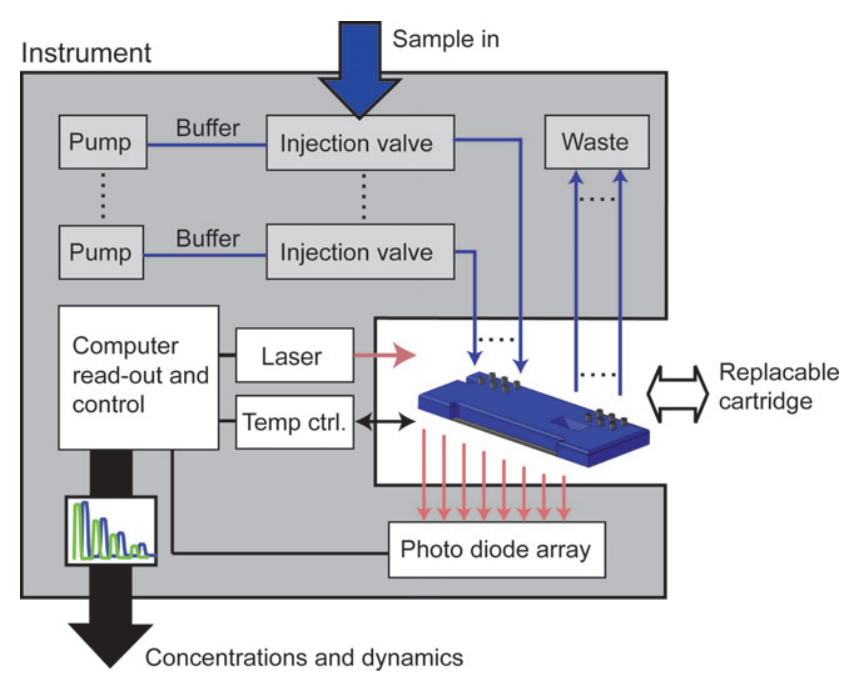

Fig. 4 A block diagram of the complete measurement system. Liquid flow is maintained by syringe pumps and the samples are injected into the flow by in-line injections valves. Different sensors are individually addressed by separate microfluidic channels. Laser wavelength tuning and signal read-out are automated by computer control. The temperature of the interchangeable cartridge can be controlled by a Peltier heat pump in the alignment platform.

chip inside the cartridge, the alignment is good enough after manual cartridge replacement to allow automatic optimisation of the light coupling by two dimensional translation of the alignment platform. After passing through the chip, light is collected from the diced chip edge by a lens focusing the eight outputs onto a linear photodiode array (XLIN-1.9-016-TE0, Xenics, Belgium) — each output onto a single pixel. ${ }^{24}$ The laser wavelength sweep and signal read-out are automated by computer control.

The temperature of the sensor cartridge can be controlled by a Peltier heat pump in the alignment platform. Since the silicon chip is in direct thermal contact with the alignment platform, effective heat exchange with the transducer sites is ensured. For applications requiring a stable temperature, the temperature of the chip can be kept within $0.1 \mathrm{~K}$. However, in the characterisation experiments presented here, no active thermal control was employed, but thermal drift compensated using on-chip referencing.

\section{Measurement principle}

The ring resonator is a refractive index to wavelength transducer. Hence, a change in refractive index, of the volume probed by the evanescent field of the optical wave circulating in the ring, shifts the spectral position of the ring resonance wavelengths. In a biosensor, this refractive index shift is caused either by a refractive index change of the bulk sample or by mass accumulation on the sensor surface. Thus, to perform a multiplex real-time label-free assay, the resonance wavelength of each ring must be tracked.

To track the resonance wavelength in all the rings at once, we continuously sweep the input wavelength by tuning the laser, splitting the light on-chip, and collecting the transmission spectra of each ring with a dedicated pixel of a photodiode array. Thus, the spectral resolution of the measurement can be adjusted by changing the wavelength step of the laser (down to a minimum step of $1 \mathrm{pm}$ for our laser). Since the ring transmission spectrum is periodic, it suffices to scan a wavelength range of just above one period, or one free spectral range (FSR), in optical terminology. Fig. S3,†shows a superposition of the concurrently collected spectra of all 6 measurement channels operating in phosphate buffered saline (PBS).

The most relevant figure for quantitative comparison of different resonant sensor systems is the obtainable detection limit:

$$
L=\frac{R}{S}
$$

where $S$ is the sensitivity, expressed as resonance wavelength shift per refractive index or mass unit, and $R$ is the sensor resolution that is the smallest spectral shift that can be measured. The sensor resolution depends on the spectral resolution of the measurement and on the total system noise. ${ }^{9}$

Our use of slot waveguides instead of conventional strip waveguides lowers the detection limit by increasing $S$. Furthermore, we employ two strategies to reduce $R$. First, on-chip references enable us to reduce the noise term of $R$, by compensating for external disturbances such as ambient temperature variations. Second, to extract the resonance wavelength, we fit an analytical model ${ }^{25}$ to the whole measured spectrum. Since the model uses information from the whole spectrum, the effective spectral noise term contributing to $R$ can be reduced well below the wavelength step of the laser. ${ }^{2}$

\section{Experiments}

\section{Volume sensing}

In a first experiment, we determined the volume refractive index sensitivity and limit of detection of the sensor chip, by injecting a dilution series of ethanol (Etanol Aa 99,7\% Eur.Ph., Solveco, Sweden) and methanol (VLSI selectipur, Merck, Germany) plugs into a running buffer of deionised (DI) water. Table 1 lists the refractive index shift, compared to pure water, for the different concentrations used. ${ }^{26}$ Note that even though pure methanol has a lower refractive index than water, dilute solutions have a higher index. The volume of the sample plugs was $100 \mu \mathrm{l}$ and the flow rate was set to $15 \mu \mathrm{min}^{-1}$. Real-time multiplex operation was demonstrated by concurrently measuring, ethanol and methanol dilutions in channels M1 and M2, respectively, while channels M3 and M4 contained still standing DI water, and served as references to monitor any drift of the DI water baseline. In a second part of the

Table 1 Refractive index solutions

\begin{tabular}{lll}
\hline & Mass percentage $^{a}$ & Refractive index shift $^{b}$ \\
\hline Ethanol & 5.97 & 0.0037 \\
& 3.98 & 0.0024 \\
& 1.99 & 0.0012 \\
& 0.994 & 0.0006 \\
Methanol & 0.500 & 0.0003 \\
& 20.1 & 0.0051 \\
& 14.3 & 0.0036 \\
& 9.82 & 0.0023 \\
& 5.00 & 0.0011
\end{tabular}

${ }^{a}$ Mass percentage (mass of solute/total mass of solution) of the injected sample solutions. ${ }^{b}$ The corresponding shift from pure water refractive index. 
experiment, the roles of M1 and M2 were interchanged to quantify the sensitivity difference between two sensors on the same chip.

\section{Surface sensing}

In a second cartridge, we studied the surface mass sensing performance by measuring the binding of anti-BSA to a chemically activated waveguide surface. Before the assembly, the $\mathrm{Si}_{3} \mathrm{~N}_{4}$ waveguides were selectively activated by a layer of the molecular linker glutaraldehyde. ${ }^{12}$ Increasing concentrations of anti-BSA were then injected in a running buffer of phosphate buffered saline (PBS) and the resonance shift monitored until saturation. The volume of the sample plug was kept at $100 \mu \mathrm{l}$, but, to better resolve the binding dynamics on the ring, the flow rate was reduced to $10 \mu 1 \mathrm{~min}^{-1}$.

In order to accurately determine the surface density of a monolayer anti-BSA on a glutaraldehyde surface, an identical binding experiment was performed using dual polarisation Young interferometry (AnaLight 4D, Farfield Scientific, Crewe, UK). ${ }^{27,28}$

\section{Measurement results}

\section{Volume sensing}

Fig. 5(A) shows the resonance wavelengths of transducers M1M4, as a functions of time, during injections in M1 and M2. As expected, the resonance wavelengths of M1 and M2 are red shifted, that is towards longer wavelengths, during injection of the higher refractive index samples and then return to baseline as the flow returns to DI water. Furthermore, the magnitude of the shift correlates with the concentration injected.

We notice a slight upward drift of the M1 and M2 baselines, during the initial repeated high concentration injections. The same trend is also visible in M3, but absent in M4. We attribute this drift to the known organic solvent absorption of PDMS. ${ }^{29}$ During injection, solvent is absorbed, and when the flow is switched back to DI, a fraction diffuses out again. This accumulation of solvent gradually lifts the baseline. Since M3 has no flow, the accumulation is more pronounced there. M4, in contrast, is unaffected, since M3 shields it off from the injected channels, and thus M4 still provides a useful reference for other disturbances, such as temperature variation. Indeed, M4 blue shifts $100 \mathrm{pm}$ during the first hour, but then stabilises and blue shifts $20 \mathrm{pm}$ during the following $2 \mathrm{~h}$. We attribute the initial transient to temperature variation caused by the start of a buffer flow in M1 and M2.

To quantify the refractive index sensitivity, we first compensate channels M1 and M2 for the drift observed in M4. The compensated time trace of the resonance wavelength shift of M1 and M2, during the injections of decreasing concentration, is shown in Fig. 5(B). The remaining baseline red shift of $50 \mathrm{pm}$ in $100 \mathrm{~min}$ is thus due only to solvent accumulation.

The volume refractive index sensitivity can now be determined by plotting the measured resonance wavelength shift as a function of the refractive index shift of the solutions injected. The slope of this plot, shown in Fig. 6, is the sensitivity. M1 and M2 show an equal refractive index sensitivity of $S_{\mathrm{n}}=246 \mathrm{~nm}$ per RIU. This transducer independence demonstrates the transducer-to-transducer repeatability of the ring resonator fabrication process.
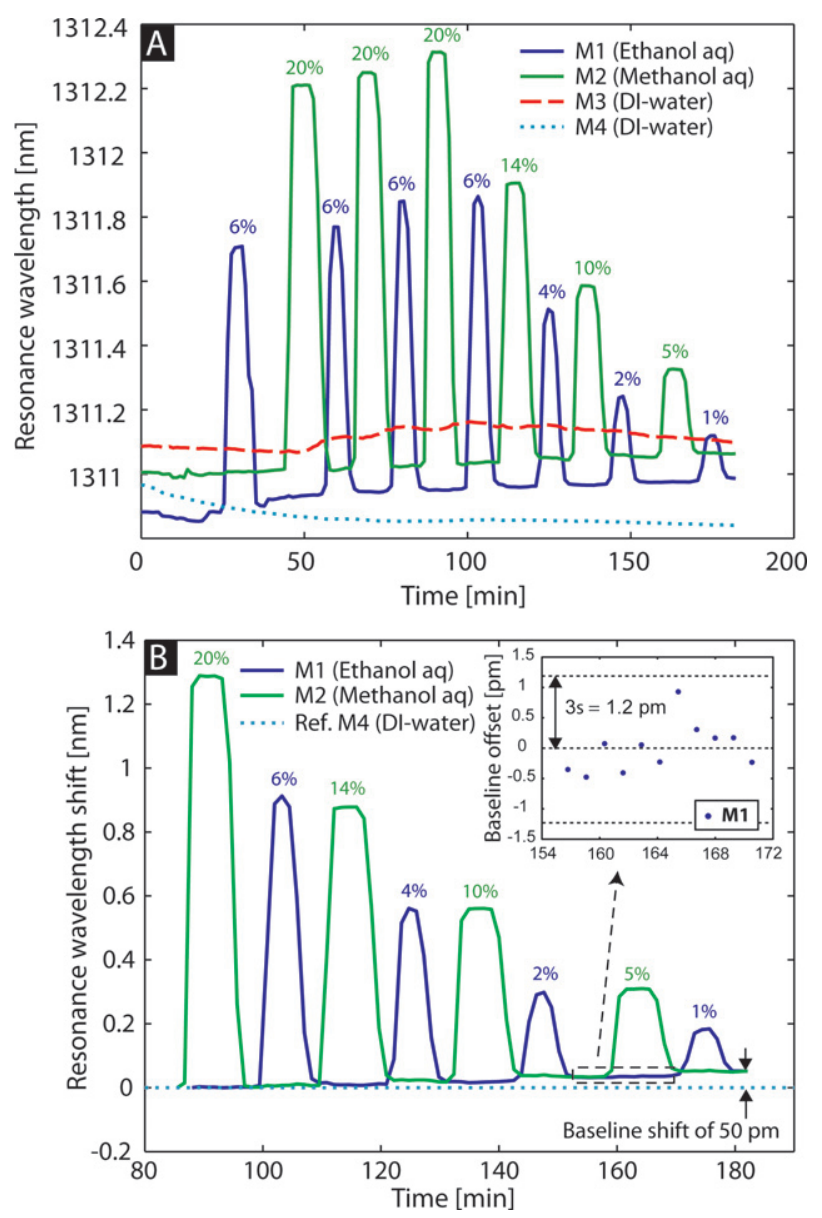

Fig. 5 (A) Resonance wavelengths of transducers M1-M4 during ethanol and methanol injections into a running buffer of DI water in channels M1 and M2, respectively. Channels M3 and M4 contain still standing DI water for reference. (B) The resonance wavelength shift of transducers M1 and M2 during ethanol and methanol injections, compensated for temperature drift by using M4 (DI water) as reference.

The inset in Fig. 5(B) shows a magnification of the measured baseline noise. We follow the convention of using 3 standard deviations $\sigma$ of the total system noise as a measure of the sensor resolution: ${ }^{9}$

$$
R=3 \sigma=1.2 \mathrm{pm}
$$

The volume refractive index detection limit is given by

$$
L_{\mathrm{n}}=\frac{R}{S_{\mathrm{n}}}
$$

and thus

$$
L_{\mathrm{n}}=5 \times 10^{-6} \mathrm{RIU}
$$

\section{Surface sensing}

Fig. 7(A) shows the resonance wavelength, as a function of time, during binding of anti-BSA to an activated waveguide surface. Increasing concentrations, from $0.061 \mu \mathrm{g} \mathrm{ml}^{-1}$ to $400 \mu \mathrm{g} \mathrm{ml}^{-1}$, are 


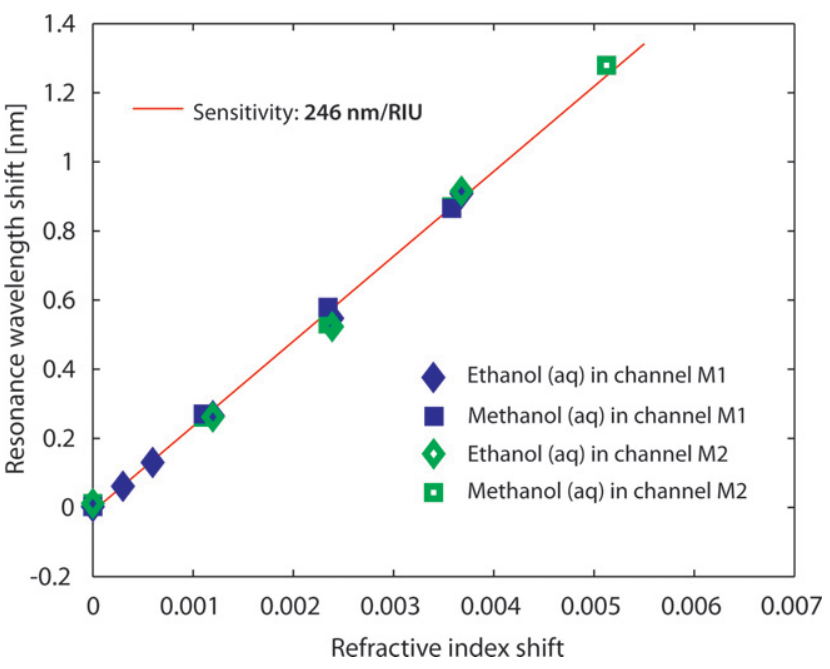

Fig. 6 Resonance wavelength shift as a function of refractive index of the solutions. Diamonds correspond to ethanol and squares to methanol injections. Filled markers correspond to injections in M1 and open markers to injections in M2. The sensors exhibit a refractive index sensitivity of $246 \mathrm{~nm}$ per RIU.

injected. The time of each injection is indicated in the plot by a filled triangle. At a concentration of $0.125 \mu \mathrm{g} \mathrm{ml}^{-1}$, a shift is clearly visible and at concentrations exceeding $50 \mu \mathrm{g} \mathrm{ml}^{-1}$ we observe removal of non-covalently bound material after each injection, indicating that the surface is saturating.

The resonance shift as a function of anti-BSA concentration, shown in Fig. 7(B), fits well to a typical sigmoid curve for binding site limited reactions, and we can estimate the shift in resonance wavelength at saturation, $\Delta \lambda=2.55 \mathrm{~nm}$, from the curve. The sensitivity for surface mass detection is given by $S_{\mathrm{m}}=\Delta \lambda / \sigma_{\mathrm{p}}$, where $\sigma_{\mathrm{p}}$ is the surface density of a molecular monolayer. The surface density of a monolayer of anti-BSA measured using dual polarisation interferometry with the Farfield AnaLight 4D system was $\sigma_{\mathrm{p}}=2.0 \mathrm{ng} \mathrm{mm}^{-2}$. This figure is slightly higher than that calculated from a monolayer using the theoretical molecular footprint for anti-BSA of $12 \times 12 \mathrm{~nm}^{2}$ indicating high surface loading. ${ }^{12}$ Using the value of the resonance shift from this experiment, we calculate a mass sensitivity of $S_{\mathrm{m}}=1.3 \mathrm{~nm} \mathrm{ng}^{-1} \mathrm{~mm}^{-2}$. Since the surface mass detection limit is given by

$$
L_{\mathrm{m}}=\frac{R}{S_{\mathrm{m}}}
$$

where $R$ is the sensor resolution, we obtain a surface mass detection limit of

$$
L_{\mathrm{m}}=0.9 \mathrm{pg} \mathrm{mm}^{-2}
$$

\section{Discussion}

The obtained volume sensing detection limit of $5 \times 10^{-6}$ RIU compares favourably both to our own previous experiments with silicon nitride slot-waveguide ring resonators ${ }^{11}$ and to other published ring resonator results. ${ }^{3,4}$ The improvement over our previously published detection limit of $2.3 \times 10^{-4} \mathrm{RIU}$ is mainly due to the significant reduction of system noise down to $1.2 \mathrm{pm}$,
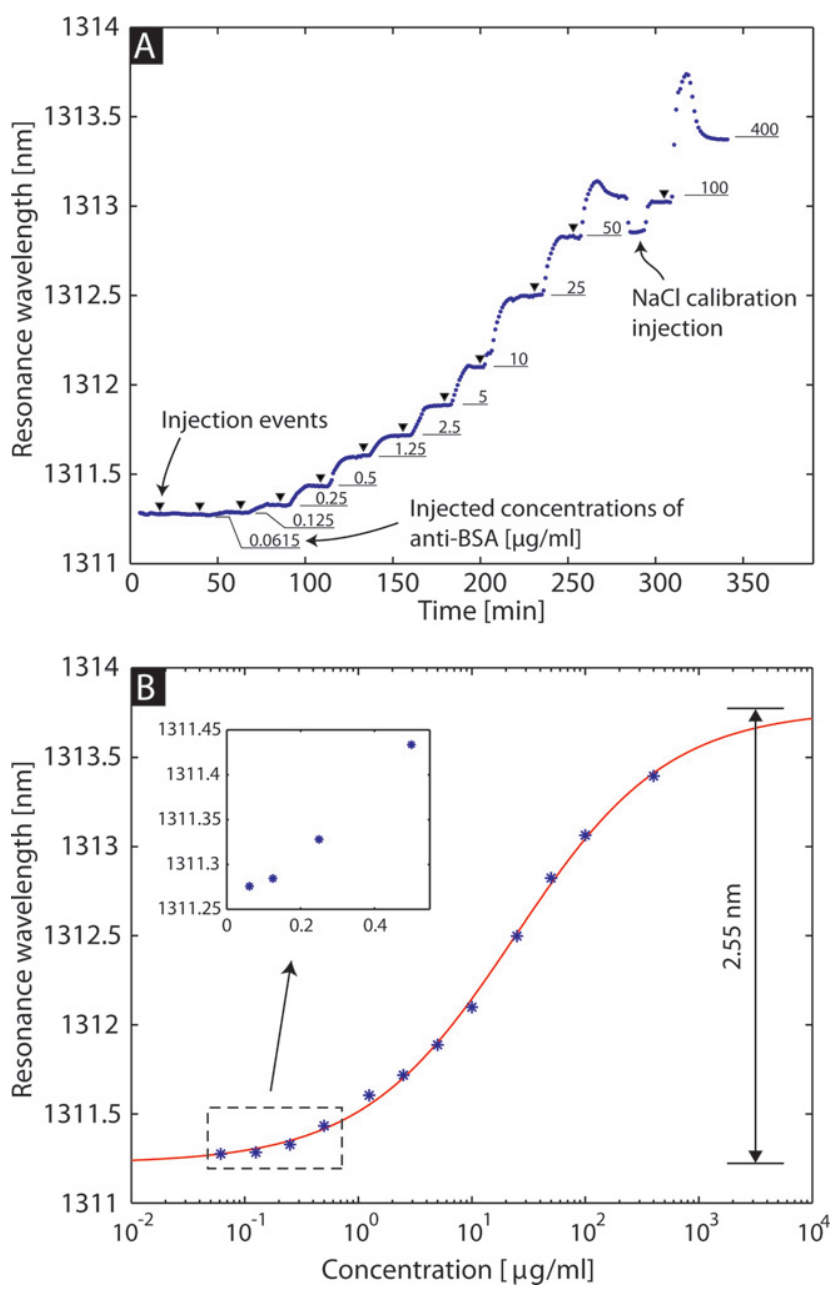

Fig. 7 (A) The time trace of increasing concentrations of anti-BSA binding to the glutaraldehyde coated transducer. Injection times are marked with filled triangles and the injected concentration noted at the plateau level. (B) The resonance wavelength as a function of injected antiBSA concentration. The solid curve is a fitted sigmoid model. The inset shows the first four points on a linear concentration scale.

achieved by using on chip temperature references, a smaller laser wavelength step, and, in particular, by fitting an analytical model to the whole spectrum obtained, thus effectively utilising all the information available. The slight increase in sensitivity, from $212 \mathrm{~nm}$ per RIU to $248 \mathrm{~nm}$ per RIU, also contributes, but to a much smaller extent. The increased sensitivity is likely due to alterations of the fabrication scheme of the ring resonators between experiments, but has not been studied in detail.

The improvement over other previously published results, such as $1.8 \times 10^{-5}$ RIU for a vertically coupled glass ring resonator ${ }^{3}$ and $1 \times 10^{-5}$ RIU for a silicon-on-insulator (SOI) ring resonator, ${ }^{4}$ is mainly due to the higher sensitivity of the slot-waveguide to volume refractive index variations.

In terms of surface sensing, the obtained detection limit of $0.9 \mathrm{pg} \mathrm{mm}^{-2}$ presents a significant improvement of our own previously published value of $28 \mathrm{pg} \mathrm{mm}^{-2}$. As is the case with volume sensing, the improvement is mainly due to the reduced system noise. Comparison with the recently published value of $17 \mathrm{pg} \mathrm{mm}^{-2}$ for an SOI ring resonator ${ }^{30}$ in a system with 
a comparable noise level of 2 pm leads us to conclude that slot waveguides also present a sensitivity advantage for surface sensing. Commercial surface plasmon resonance (SPR) biosensors based on bulk optical components have a detection limit between $1 \times 10^{-6}$ and $1 \times 10^{-7}$ RIU and a mass surface density detection limit around $1 \mathrm{pg} \mathrm{mm}^{-2}$, which is comparable to our lab-on-a-chip solution. ${ }^{16}$

The packaging method with the dual sided adhesive tape was successful and the design goals of the cartridge were reached in terms of quick leak-tight assembly up to 1 bar of pressure and easy alignment in the read-out instrument. The dual surfaceenergy adhesive mated the PDMS rubber and hard plastic shell, without the need for o-rings and without leakage between the 12 tightly spaced (3 mm apart) inlet and outlet holes. This was also the main advantage of the adhesive bonding method compared to clamping. Alignment of the cartridge in the readout instrument was efficient except that on some cartridges the PDMS protruded over the optics chip and blocked perfect alignment. This was obviously due to the manual cutting of the PDMS which was the only component which was not precision machined. Air bubbles accidentally introduced in the cartridge during change of buffer liquids, travelled through the system without getting stuck, probably due to the consistent use of smooth corners in the fluidic design.

Not all liquids can be run in the cartridge without producing artifacts in the measurement read-out. It is well known that PDMS absorbs certain organic solvents. ${ }^{29}$ For calibration purposes we decided to use concentrations of ethanol and methanol because they are absorbed little compared to other solvents. By running a reference channel with DI water sufficiently far away from the solvent filled channels, unaffected by the solvent, we could distinguish and quantify the solvent diffusion from other external influences such as temperature. This would not have been possible without on-chip references, since we would not know if it was temperature or solvent diffusion that displaced the baseline. To ensure that we would not overestimate the performance of the sensor because of the small drift caused by solvent diffusing out of the PDMS we measured the resonant shifts from the baseline after the injections, and not before.

The high sensitivity of the transducers also made it possible to detect a small shift of the baseline when changing the salt concentration of the running buffer. We believe this is due to ion diffusion into the bottom oxide cladding under the ring resonator. The drift stabilises in a few minutes but occurs again if a higher salt concentration is injected. This drift, however, did not affect the surface sensing experiments, since care was taken to match the salt concentration of the running buffer and the injected samples. The matching also avoids jumps in the resonance wavelength due to bulk refractive index change. Since the silicon nitride used in the waveguides is a good diffusion barrier, a way to avoid this problem would be to leave a thin pedestal of silicon nitride in the device layer etch step to separate the bottom cladding completely from the running buffer.

The cartridge can be functionalised either using in-line functionalisation, such as immobilising anti-BSA on the waveguides, or by spotting the biomolecule receptors directly on the transducer before cartridge assembly. In-line functionalisation is time consuming, and thus not suited for industrial screening, but is more flexible since each chip can be customised right before the experiment.
The light coupling scheme provided enough light through each of the 6 sensing channels on the chip to track the resonance wavelength. In combination with the good alignment tolerance, these results prove the suitability of using surface grating couplers to couple light into user replaceable lab-on-a-chip cartridges. Furthermore, since the detection limit of the ring resonators is not directly affected by the quality of the output beam, in contrast to e.g. Young waveguide interferometers, ${ }^{31}$ the output edge of the optical chips needs no polishing and thus the chips can be mass manufactured at low cost.

\section{Conclusions}

We have presented the design, fabrication, and characterisation of a packaged array of optical refractive index sensors, integrated with microfluidic sample handling in a compact cartridge. Our novel packaging method allows fast and leak-tight assembly of cartridges with multiple tightly spaced fluidic interconnects.

We have determined both the volume and the surface sensing limit of detection. Using the multiple transducers available on the chip, we separated and compensated for different kinds of external disturbances, resulting in much improved noise level, compared to our previously published results. This improvement, combined with the high sensitivity of the slot-waveguide ring resonators, yields a volume refractive index detection limit of $5 \times 10^{-6}$ RIU and surface mass detection limit of $0.9 \mathrm{pg} \mathrm{mm}^{-2}$, to our knowledge the best reported values for integrated ring resonator sensors.

\section{Acknowledgements}

This work was financed by the European Commission through the sixth framework program FP6-IST-SABIO (026554). K. B. Gylfason acknowledges support of the Steinmaur Foundation, Liechtenstein.

\section{Notes and references}

1 D. Janasek, J. Franzke and A. Manz, Nature, 2006, 442, 374-380.

$2 \mathrm{H}$. Sohlström and M. Öberg, The 8th European Conference on Integrated Optics, Optical Society of America, Washington DC, USA, 1997, pp. 322-325.

3 A. Yalcin, K. C. Popat, J. C. Aldridge, T. A. Desai, J. Hryniewicz, N. Chbouki, B. E. Little, O. King, V. Van, S. Chu, D. Gill, M. Anthes-Washburn, M. S. Unlu and B. B. Goldberg, IEEE J. Sel. Top. Quantum Electron., 2006, 12, 148-155.

4 K. De Vos, I. Bartolozzi, E. Schacht, P. Bienstman and R. Baets, Opt. Express, 2007, 15, 7610-7615.

5 A. Ramachandran, S. Wang, J. Clarke, S. Ja, D. Goad, L. Wald, E. Flood, E. Knobbe, J. Hryniewicz, S. Chu, D. Gill, W. Chen, O. King and B. Little, Biosens. Bioelectron., 2008, 23, 939-944.

6 J. A. De Feijter, J. Benjamins and F. A. Veer, Biopolymers, 1978, 17, $1759-1772$.

7 A. Ksendzov and Y. Lin, Opt. Lett., 2005, 30, 3344-3346.

8 I. M. White, H. Oveys, X. Fan, T. L. Smith and J. Zhang, Appl. Phys. Lett., 2006, 89, 191106.

9 I. M. White and X. Fan, Opt. Express, 2008, 16, 1020-1028.

10 V. R. Almeida, Q. Xu, C. A. Barrios and M. Lipson, Opt. Lett., 2004, 29, 1209-1211.

11 C. A. Barrios, K. B. Gylfason, B. Sánchez, A. Griol, H. Sohlström, M. Holgado and R. Casquel, Opt. Lett., 2007, 32, 3080-3082.

12 C. A. Barrios, M. J. Bañuls, V. González-Pedro, K. B. Gylfason, B. Sánchez, A. Griol, A. Maquieira, H. Sohlström, M. Holgado and R. Casquel, Opt. Lett., 2008, 33, 708-710.

13 T. Gervais and K. F. Jensen, Chem. Eng. Sci., 2006, 61, 1102-1121. 
14 Y. Xia and G. M. Whitesides, Angew. Chem., Int. Ed., 1998, 37, 550-575.

15 D. C. Duffy, O. J. Schueller, S. T. Brittain and G. M. Whitesides, J. Micromech. Microeng., 1999, 9, 211-217.

16 J. C. Lotters, W. Olthuis, P. H. Veltink and P. Bergveld, J. Micromech. Microeng., 1997, 7, 145-147.

17 W. W. Y. Chow, K. F. Lei, G. Shi, W. J. Li and Q. Huang, Smart Mater. Struct., 2006, 15, S112-S116.

18 J. Kim, R. Surapaneni and B. K. Gale, Lab Chip, 2009, 9, 1290-1293.

19 G. Maire, L. Vivien, G. Sattler, A. Kaźmierczak, B. Sánchez, K. B. Gylfason, A. Griol, D. Marris-Morini, E. Cassan, D. Giannone, H. Sohlström and D. Hill, Opt. Express, 2008, 16, 328-333.

20 S. Wolf and R. N. Tauber, Silicon Processing for the VLSI Era, Vol. 1: Process Technology, Lattice Press, Sunset Beach CA, USA, 1999.

21 J. M. Heaton, R. M. Jenkins, D. R. Wight, J. T. Parker, J. C. H. Birbeck and K. P. Hilton, Appl. Phys. Lett., 1992, 61, 1754-1756.

22 P. Dumon, W. Bogaerts, V. Wiaux, J. Wouters, S. Beckx, J. Van Campenhout, D. Taillaert, B. Luyssaert, P. Bienstman, D. Van Thourhout and R. Baets, IEEE Photon. Tech. Lett., 2004, 16, 1328-1330.

23 Y. Shani, C. H. Henry, R. C. Kistler, K. J. Orlowsky and D. A. Ackerman, Appl. Phys. Lett., 1989, 55, 2389-2391.
24 A. Kaźmierczak, F. Dortu, O. Schrevens, D. Giannone, L. Vivien, D. M. Morini, D. Bouville, E. Cassan, K. B. Gylfason, H. Sohlström, B. Sánchez, A. Griol and D. Hill, Opt. Eng., 2009, 48, 14401.

25 A. Yariv, Electron. Lett., 2000, 36, 321-322.

26 CRC Handbook of Chemistry and Physics, ed. D. R. Lide, CRC; Taylor \& Francis distributor, London, UK, 2008.

27 G. H. Cross, A. A. Reeves, S. Brand, J. F. Popplewell, L. L. Peel, M. J. Swann and N. J. Freeman, Biosens. Bioelectron., 2003, 19, 383-390.

28 G. H. Cross, N. J. Freeman and M. J. Swann, in Dual Polarization Interferometry: A Real-Time Optical Technique for Measuring (Bio)Molecular Orientation, Structure and Function at the Solid Liquid Interface, in Handbook of Biosensors and Biochips, ed. R. S. Marks, Wiley, 2007, vol. 1, ch. 32, pp. 549-568.

29 J. N. Lee, C. Park and G. M. Whitesides, Anal. Chem., 2003, 75, 6544-6554.

30 K. De Vos, J. Girones, S. Popelka, E. Schacht, R. Baets and P. Bienstman, Biosens. Bioelectron., 2009, 24, 2528-2533.

31 A. Ymeti, J. Greve, P. V. Lambeck, T. Wink, S. W. F. M. Vanhovell, T. A. M. Beumer, R. R. Wijn, R. G. Heideman, V. Subramaniam and J. S. Kanger, Nano Lett., 2007, 7, 394-397. 